\title{
Semantic-Based Image Retrieval in Remote Sensing Archive: An Ontology Approach
}

\author{
Ning RUAN \\ School of Computer Science \\ and Engineering \\ Beihang University \\ Beijing, China \\ Email: ruanning@cse.buaa.edu.cn
}

\author{
Ning HUANG \\ School of Computer Science \\ and Engineering \\ Beihang University \\ Beijing, China \\ Email: hn@buaa.edu.cn
}

\author{
Wen HONG \\ Institute of Electronics \\ Chinese Academy of Sciences \\ Beijing, China \\ Email: whong@mail.ie.ac.cn
}

\begin{abstract}
In recent years, remote sensing image data have increased significantly due to the improvement of remote sensing technique. On the other hand, data acquisition rate will also be accelerated by increasing satellite sensors. Hence, it is a large challenge to make full use of so considerable data by conventional retrieval approach. The lack of semantic based retrieval capability has impeded application of remote sensing data. To address the issue, we propose a framework based on domain-dependent ontology to perform semantic retrieval in image archives. Firstly, primitive features expressed by color and texture are extracted to gain homogeneous region by means of our unsupervised algorithm. The homogeneous regions are described by high-level concepts depicted and organized by domain specific ontology. Interactive learning technique is employed to associate regions and high-level concepts. These associations are used to perform querying task. Additionally, a reasoning mechanism over ontology integrating an inference engine is discussed. It enables the capability of semantic query in archives by mining the interrelationships among domain concepts and their properties to satisfy users' requirements. In our framework, ontology is used to provide a sharable and reusable concept set as infrastructure for high level extension such as reasoning. Finally, preliminary results are present and future work is also discussed.
\end{abstract}

Keywords- Image retrieval; Ontology; Semantic reasoning; Relevance feedback

\section{INTRODUCTION}

Remote sensing images acquired by increasing satellite sensors have increased enormously over the last few years. How to use so huge data efficiently has become an important issue to us. At present, the accessibility of the data is limited to queries on time of acquisition, geographical coordinates, sensor type and acquisition mode [1]. Such a limitation constraints the usability of the images, so only few of the acquired image data can actually be used. In the future, the access to image archives will even become more difficult due to the enormous data quantity acquired by a new generation of high-resolution satellite sensors [2]. To overcome such a challenge problem, new technologies those allow the users to access remote sensing images based on semantics are required.

During past few years, Content-based Image Retrieval (CBIR) technique has been proposed to overcome the obstacles of text or keyword-based approach methods. At that time, many research efforts were made on CBIR technique and many CBIR systems were developed. IBM QBIC is one of the earliest commercial image retrieval systems, while MIT Photobook is one of the earliest ones in the academic domain. Others include Berkeley Blobworld, Columbia VisulSeek et al [1]. Almost all of systems query the data by their visual similarity with respect to low level features of images, e.g. color, texture, and shape. These conventional CBIR systems utilizing low level features are still beyond semantic similarity. Therefore, there is still a wide gap between image and human's interpretation.

In recent years, many researchers devote to extend image retrieval methodologies beyond low-level characteristics to semantic. Relevant interaction and feedback method is proposed to extract semantic from images in [3]. In [4], an integrated probabilistic image semantic description multi-level model named IPSM (Image Probability Semantic Model) is presented. Its experimental result shows that it is effective in characterizing image high-level semantic content. In remote sensing domain, Knowledge Driven Information Mining in Remote Sensing Image Archives (KIM) [2] system is implemented by German Aerospace Center (DLR). In the KIM system, it puts forward an innovated image description method that using basic characteristics of image pixels or region as representative of image information. Based on it, knowledge discovery and data mining with hierarchical segmentation has been proposed. Through Bayesian Network and interactive learning technique, the system provides capabilities to explore the intrinsic prosperities of a region to develop heuristics for an automatic labeling of image regions.

By far, there are two drawbacks in current retrieval systems [5]. Firstly, semantic extracted from images is just defined by individuals which can not been shared by other users in the similar community. It induces diverse understandings of the same object and interrupts the sharing of knowledge. Following that, lacking an effective method to describe and utilize the relationships among concepts which is most important for image interpretation, so system can not understand users' intention better. For example, one may define the object of image as "Lake", but others can not find it when someone searches "Water" in the image archives.

To address the limitation of current knowledge mining 
system, we put forward a framework that integrates ontology technique, reasoning over ontology method and relevance feedback technique to carry out retrieval task. We will show how ontologies can be helpful for users in formulating the information need, the query and the answers. Main characteristics of proposed framework are: 1) advancing a means to represent sharable and reusable domain knowledge by ontology; 2) providing several query facilities including semantic retrieval to obtain information from image data to higher semantic concepts.

\section{System ARChitecture}

According to the framework shown in fig. 1, the processing procedure is composed of 3 levels: Feature Extraction, Semantic Mapping and Higher Conceptual Description. Lowlevel features are extracted and regions are indexed by them at Feature Extraction Level. These regions are machinecentered and the association with meaningful semantic concept is required. To achieve the goal, a set of domain specific concepts is developed to describe the content of region by user's interaction. Following that, the concepts are organized and relationships among these concepts are also defined at Higher Conceptual Level.

As shown in fig. 1, the procedure starts with the primitive feature extraction. Low-level features are extracted from raw data and stored in feature database. To reduce computational complexity and facilitate the user interactive learning, these low-level features are classified into feature classes by an unsupervised classification. These classes are considered as metadata and conserved in the knowledge base. Through these classes, regions of image are well indexed by low-level features. In the actual application, user will access images from various perspectives. At the higher conceptual level, the domain specific ontology stored in knowledge base is constructed to depict the domain concepts and their relationships. We employ Ontology Web Language (OWL) [6] to build ontologies. When higher conceptual description and lower level features description are prepared, an effective means is needed to link these two parts and implement the complete processing procedure. The learning phase is applied in this stage. Interactive learning is carried out by means of relevance feedback technique based on Bayesian Learning [7]. Users can train the domain concepts by selecting positive and negative region through mouse's left or right clicking. It enables user refine the semantic relationships between image regions and semantic concepts iteratively. The details of these works are discussed in [7].

The system provides various query means to advance search capability. Users can choose any of them which suit for application. During querying, user interacts with system based on a friendly interface. The interface is designed as a Java Applet, so users can access it through any web browser conveniently. The system supports both query by basic properties (such as sensor type or acquisition time) and query by region example. Furthermore, we also propose a design which makes our system more intelligent in attempt to bridge the semantic gap.

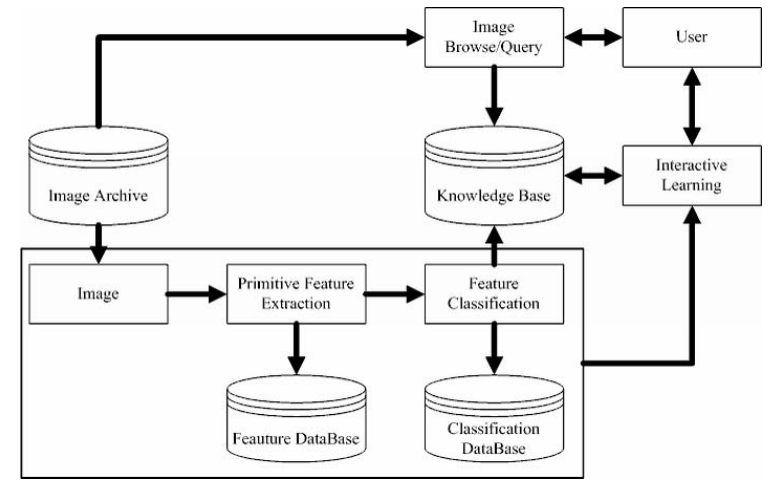

Fig. 1. Proposed framework depicting the overall processing procedure

Users can retrieval images by choosing interested semantic concepts which have been trained. An inference approach over ontology is used in this step. Based on reasoner and knowledge base, we perform semantic reasoning to improve understanding of users' requirements at system level. The appropriate semantic descriptor for users' requirements is retrieved and used to carry out query task. Finally, querying result ranked by probability and positive percentage returns as a web page.

\section{Feature Extraction AND Classification}

Remote sensing image mainly contains 3 primitive features: color, texture and shape. Shape is a structural feature which is difficult to extract. Therefore, we combine texture and color to describe remote sensing image. In this paper, Haralick's gray level co-occurrence matrices method [8] is used to extract textural information. The gray level co-occurrence matrix is the two dimensional matrix of joint probabilities $[P(i, j, d, \theta)]$ between pairs of pixels (one with gray level $i$ and the other with gray level $j$ ), separated by a distance $d$, in a given direction $\theta$. In order to represent textural information contained in gray level co-occurrence matrix, 4 feature parameters are defined. We compute this feature for 1 to 5 pixel distances and for $0^{\circ}, 45^{\circ}, 90^{\circ}, 135^{\circ}$ orientations. This constitutes a 20 dimensional feature vector. In addition to optical image, we also consider color characteristic as feature. In our work, color feature vector is defined as $\left\{m_{R} / m_{G}, m_{B} / m_{G}\right\}$ where being the sample mean of $i(i=R, G, B)$ plane respectively. We do so just take the illumination influence into consideration.

Though texture and color feature extraction, 22-dimensional feature is gained by combining 20-dimensional texture feature and 2-dimensional color feature. Therefore, some clustering methods can be used to obtain feature classes, such as Bayesian classification, K-Means et al. In our work, we adopt an unsupervised K-Means classifier to achieve it. K-Means is a dynamic clustering algorithm, that is, cluster center will be changed during clustering. In order to make full use of K-Means algorithm, one precondition is to determine the optimal classes number while it is very difficult. We propose an approach to solve the problem during clustering. Equation (1) calculates the mean of samples in a given classification. 
As (2) shows, $J_{e}$ is an evaluation function used to calculate error sum of squares for a special classification.

$$
\begin{gathered}
m_{i}=\frac{1}{N_{i}} \sum_{y \in \omega_{i}} y \\
J_{e}=\sum_{i=1}^{k} \sum_{y \in \omega_{i}}\left(y-m_{i}\right)^{2}
\end{gathered}
$$

We give a possible classes number list and each one represents classes number in a classification. For every possible classes number $e$ in the list, we calculate $J_{e}$. Following that, a curve is drew based on $e$ (as $\mathrm{X}$ axis) and $J_{e}$ (as $\mathrm{Y}$ axis), then find out turning point of the curve, that is the optimal classes number.

The variables of equations are defined as follows:

- $\omega_{i}$ represents $i$-th class in a special classification.

- $N_{i}$ represents the number of the set $\omega_{i}$

- $m_{i}$ represents the mean of the set $\omega_{i}$

- $y$ represents a sample contained in the set $\omega_{i}$

- $J_{e}$ represents the error sum of squares for a given classes number $e$

Through the method mentioned above, the whole image is segmented to various regions, that is, every pixel is marked with corresponding class number.

\section{ONTOLOGY BUILDing}

Ontology is a formal explicit specification of a shared conceptualization [9]. It provides a shared and common understanding of a domain that can be communicated across people and application systems. In a domain, ontology defines a set of representational terms that we call concepts. Interrelationships among these concepts can describe a target domain. Ontology can be built in two ways: generic and domain specific. One of main differences between generic ontology and domain specific ontology is description granularity. That is, generic ontology describes a wider world in a coarse way, while domain specific ontology is in a fine way. For our purpose, we adopt domain specific ontology. Fine-described concepts enable us to determine specific relationships among features in remote sensing images which can be used to distinguish them more effectively. We build our ontologies by using ontology develop tool (ODT) [10], a new graphical ontology editor extends the conceptual graph methodology to elicit the domain knowledge better (Screenshot of ODT is shown in Fig. 2). At the meanwhile, the capability for describing relationships among concepts is also provided in ODT. To complete semantic reasoning function, reasoner is integrated in the system. This part will be demonstrated in following Section.

We put forward "SAR Imagery" as an example to demonstrate ontology building. Fig. 3 illustrates the sample ontology for SAR imagery domain. This ontology can be obtained from generic remote sensing terminology and domain experts. The ontology is described by directed graph. Each node represents a concept which contains a unique name and several

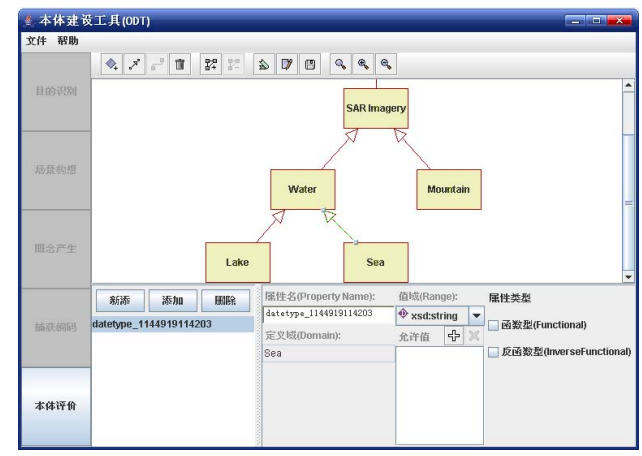

Fig. 2. Interface for ontology building tool

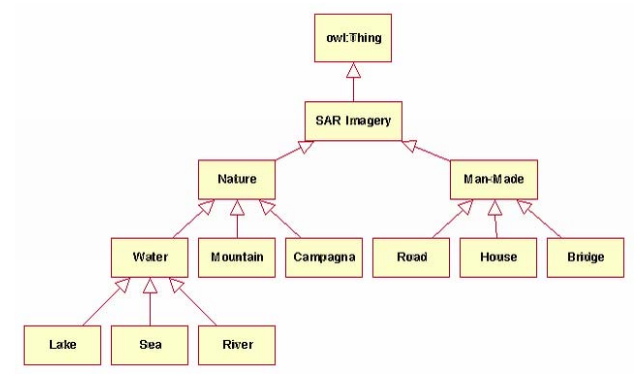

Fig. 3. An example ontology for SAR Imagery

features. Each feature describes one characteristics of an image. In ontologies, concepts are interconnected by means of interrelationship. Thus, each line with arrow shows the interrelationship between two connected nodes. Three kinds of inter-relationships are used to build our ontologies: IS-A, Instance-Of and Part-Of. Every image would be instance of one or more corresponding concepts because one image often includes many object scenes. Thus, domain knowledge is well defined and will be used to perform semantic retrieval.

\section{Semantic-based Retrieval}

Incredible growth of SAR image data and more widely application on the basis of SAR image require a new query mode which can understand users' requirement more accurately and fulfill users' needs. In our system, semantic reasoning mechanism is employed to solve this problem. On query section, users will configure some properties such as acquisition time and sensor type; most important of all, concepts in which users are interested are also selected. Note that, selected concepts are not used to perform query task directly since they can be refined to provide better understanding. Based on knowledge base which contains domain specific description for concepts and their interrelationships, reasoner mines associations among user selected concept, concepts and concepts' properties in the knowledge base. These associated concepts and their properties are combined as an integrated descriptor to depict user requirement, and the descriptor is more accurate than original concept for system in user requirement description. Our system performs semantic based retrieval task by using the descriptor mentioned above. The query results are image 


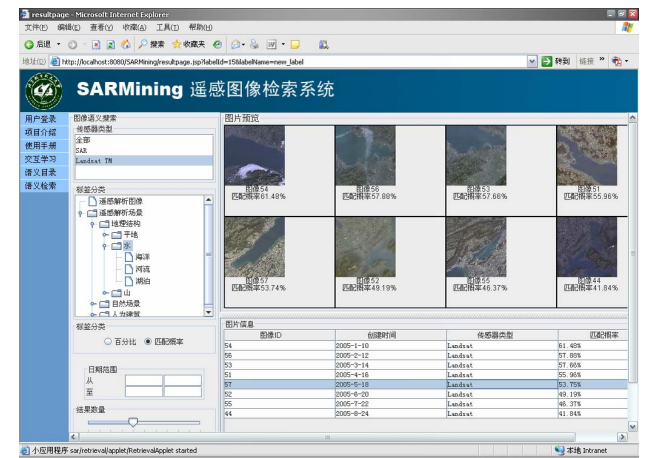

Fig. 4. Result of semantic query for "Water"

indices used to track the real image data which are stored in special image database. According to these indices, the miniature of real image will be shown in users' browser.

Recently, there are a number of widely used reasoners. On account of extension and system integration, we choose Jena [11] as our system's reasoner. Jena2 (latest version 2.3) not only includes a number of predefined reasoners, a range of inference engines or reasoners (such as FaCT, FaCT++ and Racer) are also allowed to be plugged into it. It is convenient for us to extend reasoning ability according to changes of application. Besides that, Jena is developed in Java which allows us to integrate with our system seamlessly.

We take an example to illustrate the reasoning mechanism based on example ontology described in previous section. For instance, user wants to query "Landsat TM imagery for water body". In example ontology (shown in Fig. 3), "Water" is an upper concept and has some subclasses like "sea", "lake" and so on. Though reasoning mechanism, reasoning subsystem finds out that some concepts including "Sea", "Lake" and "River" are subclass of "Water", and these concepts all inherit the "Sensor Type" property of "SAR Imagery". Therefore, our system yields the image set including "Sea", "Lake" and "River" imagery whose acquisition sensor type is "Landsat TM" as query result. This is just a simple paradigm. Along with the enrichment of complex relationships and domain concepts, semantic retrieval ability will be improved greatly if incorporated with special ontology inference engine.

\section{Preliminary Result and Future Work}

In this paper we have proposed a framework to achieve semantic-based retrieval in remote sensing archives. A new method is provided to identify the optimal classes number in feature classification. In addition, domain specific knowledge is expressed by means of ontology through our ontology development tool (ODT). Furthermore, we investigate reasoning mechanism based on ontology to enable the capability of semantic retrieval. Our system is deployed as an interactive web application and users can adjust users' requirements iteratively. A friendly retrieval interface provides an ability to query and explore information from different perspectives.

Fig. 4 depicts the retrieved images of semantic query for "Water". The images are ranked according to posterior prob- ability which means to what extend this image can be labeled by "Water". In previous domain ontology, "Sea", "Lake" and "River" are subconcepts of "Water". Therefore, retrieval result includes all querying results for sub-concepts of "Water". In order to retrieve the required images, we can click the left catalogs to choose an appropriate concept located at the center left of screen. In addition, some useful facilities are provided. Users can specify sensor type, acqusition time to satisfy their needs. As shown in Fig. 4, there is a property table under the retrieved image panel. Each item in property table represents a corresponding image in the above panel. Through property table, users can obtain detail information (such as image id, acqusition time, sensor type and so on) about retrieved images conveniently.

For future work, we will explore knowledge based feature classification to provide a more accurate segmentation in remote sensing images. Another important aspect is to improve the performance of image processing. We consider that $\mathrm{P} 2 \mathrm{P}$ (Peer-to-Peer) computing may be an available method. Also, complex semantic retrieval capability should be advanced which can incorporate with nature language processing.

\section{ACKNOWLEDGMENT}

The authors would thanks to Wang Feng and Luo Xiuchun for their excellent work in designing and implementing system. The system is also developed by Lin Zhengwei and Wu Tao. They would also like to thank Wang Jianghua, Zhao Jun, and Diao Shihan for designing and developing ODT. In particular, they would like to thank the Institute of Electronics, Chinese Academy of Sciences for their funding and cooperation, and this work is also supported by Aviation Science Foundation of China.

\section{REFERENCES}

[1] C. Chang, B. Moon, A. Acharya, C. Shock, A. Sussman, and J. Saltz, "Titan: a high-performance remote-sensing database," in Proc. IEEE International Conference on Data Engineering, Apr. 1997, pp. 375-384.

[2] M. Datcu, H. Daschiel, Apelizzari, M. Quartulli, A. Galoppo, A. Colapicchioni, M. Pastori, K. Seidel, P. G. Machetti, and S. D'Elia, "Information mining in remote sensing image archives: system concepts," IEEE Trans. Geosci. Remote Sensing, vol. 41, pp. 2923-2936, 2003.

[3] Q. Tian, P. Hong, and T. S. Huang, "Update relevant image weights for content-based retrieval using support vector machines," in Proc. IEEE International Conference on Multimedia and Expo, vol. 2, 2000, pp. 1199-1202.

[4] W. Chong-Jun, Y. Yu-Bin, and C. Shi-Fu, "Algorithms of high-level semantic-based image retrieval," Journal of Software, vol. 15, 2004.

[5] F. Wang and N. Huang, "A semantic based method for remote sensing images," Journal of Computer Research and Application, 2006.

[6] "Ontology web language(owl)." [Online]. Available: http://www.w3. org/TR/owl-features/

[7] M. Schroder, H. Rehrauer, K. Seidel, and M. Datcu, "Interactive learning and probabilistic retrieval in remote sensing image archives," IEEE Trans. Geosci. Remote Sensing, vol. 38, pp. 2288-2298, 2000.

[8] S. Aksoy and R. Haralick, "Textural features for image database retrieval," in Proc. IEEE International Workshop on Content-Based Access of Image and Video Libraries, 1998, pp. 45-49.

[9] T. Gruber, "A translation approach to portable ontology specifications," Knowledge Acquisition, vol. 5, pp. 199-220, 1993.

[10] J. Wang, "The research and implementation of ontology development method and tool," Master's thesis, Beihang University, 2006.

[11] "Jena." [Online]. Available: http://www.hpl.hp.com/semweb/jena-top. html 